\title{
COMPARISON OF BLOCK VERSUS WEEKLY UNDULATING PERIODIZATION MODELS ON ENDOCRINE AND STRENGTH CHANGES IN MALE ATHLETES
}

\author{
Sandro Bartolomei ${ }^{1,2}$, Jay R. Hoffman ${ }^{2}$, Jeffrey R. Stout ${ }^{2}$, Maddalena Zini', \\ Claudio Stefanelli ${ }^{3}$, and Franco Merni ${ }^{1}$ \\ 'Department of Biomedical and Neuromotor Science, University of Bologna, Italy \\ ${ }^{2}$ Sport and Exercise Science, University of Central Florida, Orlando, FL, USA \\ ${ }^{3}$ Department for Life Quality Studies, University of Bologna, Italy
}

Original scientific paper

UDC: 796.015.2:796.012.11-055.1

\begin{abstract}
:
The aim of this study was to examine the hormonal and strength responses to different periodization models of resistance training in male athletes. Eighteen experienced resistance trained males were randomly assigned to either a block (BP; $\mathrm{n}=10$; age: $23.7 \pm 2.9 \mathrm{yr}$; body mass: $78.5 \pm 11.3 \mathrm{~kg}$; height: $1.77 \pm 0.05 \mathrm{~m}$ ) or weekly undulating (WUP; $\mathrm{n}=8$; age: $26.0 \pm 5.7 \mathrm{y}$; body mass: $78.9 \pm 12.4 \mathrm{~kg}$; height: $1.79 \pm 0.05 \mathrm{~m}$ ) periodized resistance training program. Both programs consisted of four-training sessions per week for 15 weeks, and each was equated for training volume. Analysis of variance was used to compare strength performance and changes in hormone response between groups. Salivary samples were taken before and after the first and the last workout of each mesocycle of the training program and assessed for testosterone $(\mathrm{T})$ and cortisol (C). Maximal strength testing occurred before and after the 15-week training program. A greater increase $(\mathrm{p}=.040)$ in bench press strength was observed in BP compared to WUP, while no between group differences were noted for lower body isometric strength $(p=.168)$ and lean body mass $(p=.344)$. Significant elevations in $\mathrm{T}$ were seen in both groups following the power training phase, while no differences were noted between BP and WUP during any other training cycle. Results indicated that BP stimulated greater gains in upper body strength compared to WUP. In addition, the power phase of training may provide a greater anabolic hormone response.
\end{abstract}

Key words: program design, strength, performance, testosterone

\section{Introduction}

Periodization is a systematic planning of training in order to optimize physiological adaptations and prevent the overtraining syndrome (Buford, Rossi, Smith, \& Warren, 2007; Zatsiorsky \& Kraemer, 2006). The terminology and concepts of periodization has been proposed by Matveev (1977) and by Nádori and Granek (1989) in the early 1960s. In 1965 Matveev (1965) proposed an organization of the annual training cycle based upon cyclical undulations of training factors. Weekly Undulating Periodization (WUP), proposed by Poliquin (1988), is characterized by variations of training contents within each mesocycle and a wave-like distribution of training stimuli (Poliquin, 1988).

The block periodization (BP) model was first introduced by Verkhoshansky (1977) to reduce the total training volume in elite athletes and focus each training phase on a specific goal (Issurin,
2010). The BP model is comprised of mesocycles characterized by a concentrated training stimulus with a specific goal (Verkhoshansky, 2001). For the strength/power athlete the first phase is often focused on muscle hypertrophy and is characterized by a high volume of training at a moderate intensity in order to increase work capacity. In the second phase of training, intensity is increased and training volume is reduced to stimulate maximal strength development. The last block of training is focused on power training and is accompanied by a decrease in training volume. Several studies have compared various periodization models, (Bartolomei, Hoffman, Stout, \& Merni, 2014; Bartolomei, Stout, Fukuda, Hoffman, \& Merni, 2015; Hoffman, Wendell, Cooper, \& Kang, 2003; Stone, et al., 2000), but none have reported on hormonal changes during a training cycle. This is surprising considering that one of the primary goals of peri- 
odizing the athlete's training program is to minimize the risk of overtraining, and endocrine measures have been suggested to be a potential marker for overtraining syndrome (Fry \& Kraemer, 1997).

Differences in the distribution of workload in WUP and BP may elicit different metabolic and endocrine responses, which may lead to different adaptations. Salivary hormones are thought to reflect the concentrations of the free hormone in the blood (Umeda, et al., 1981; Vittek, L'Hommedieu, Gordon, Rappapott, \& Southren, 1985). Some studies have used saliva as a biological medium to assess the hormonal response to resistance training (Beaven, Gill, \& Cook, 2008; Cadore, et al., 2001; Kraemer, et al., 2001; Crewther, Cronin, Keogh, \& Cook, 2008) and managed to provide an advantage in understanding the endocrine response in situations in which athletes or coaches are not comfortable in providing or in allowing for blood specimens. Several studies have demonstrated validity of salivary measures of testosterone and cortisol in response to high intensity exercise (Crewther, Lowe, Ingram, \& Weatherby, 2010; McGuigan, Egan, \& Foster, 2004).

Studies examining the response of salivary hormonal changes to training programs are limited. Kraemer et al. (2001) reported that resistance training was unable to affect the circadian rhythm of salivary testosterone during a 16-hour measurement period after a single resistance training session. Similarly, Beaven et al. (2008) reported no changes in acute testosterone and cortisol concentrations following four different resistance training workouts in elite rugby players. In contrast, Crewther et al. (2008) reported significant elevation in postexercise salivary concentrations of testosterone and cortisol during an acute hypertrophy workout compared to strength or power workouts. Cadore et al. (2009) also reported increases in post-workout salivary testosterone in experienced, resistance trained men compared to untrained men. However, these studies have generally focused on the acute response to training or training program. However, no studies are known that have compared the endocrine response to prolonged training protocols in men. Thus, the purpose of this investigation was to compare the effects of weekly undulating and block periodization programs on maximal strength and hormonal changes in highly trained men.

\section{Methods}

\section{Participants}

Eighteen experienced resistance trained men participated in this study. Participants were competitive strength and power athletes competing in wrestling, rugby or throwing events of track and field. At the time of study recruitment they were engaged in resistance training at least three times per week for the past three years. During the training period, participants were not allowed to participate in any other physical activity or competition. Participants were randomly assigned to one of the two groups. Group $1(\mathrm{M} \pm \mathrm{SD} ; \mathrm{n}=8$; age: $26.0 \pm 5.7$ years; body mass: $78.9 \pm 12.4 \mathrm{~kg}$; height: $1.79 \pm 0.05 \mathrm{~m}$ ) used the Weekly Undulating Periodization program (WUP) and Group $2(\mathrm{n}=10$; age $=23.7 \pm 2.9$ years; body mass: $78.5 \pm 11.3 \mathrm{~kg}$; height: $1.77 \pm .05 \mathrm{~m})$ used the Block Periodization program (BP). All participants signed an informed consent document and the study was approved by the Alma Mater Studiorum - University of Bologna Bioethics Committee.

\section{Experimental design}

Participants were randomly divided into two experimental groups that differed in their training scheme. The first group used a weekly undulating periodized training model with a wavelike approach in regard to intensity and volume of training within each mesocycle, whereas the second group used a block periodization scheme that maintained the same training intensity and volume throughout each mesocycle. Both groups trained for 15 weeks. Assessments included anthropometrics (body mass and body composition), maximal strength of upper and lower body, and testosterone and cortisol levels in salivary samples. Anthropometric measures were conducted prior to and at the end of each mesocycle. Salivary samples were collected immediately before and after resistance training sessions. Measurements were carried out 12 times for each subject, pre and post six different training sessions (T1-T6).

\section{Resistance training protocols}

The 15-week resistance training program for each group can be seen in Table 1. All participants exercised four days per week, and the exercises performed were the same for each group. The groups differed only in the workload distribution across the training period. Although the format of intensity ( $\%$ of repetition maximum $[\mathrm{RM}]$ ) and volume (number of repetitions $\mathrm{x}$ sets $\mathrm{x} \%$ of $1 \mathrm{RM}$ ) of training differed between the groups, the two programs had an equal total training volume across the 15 weeks of training. Participants were monitored during each training session. Also, they recorded all the workloads used in a logbook, which was collected by one of the investigators following each workout. Analysis of the logbooks indicated that the participants adhered to the loads prescribed by the training program. Feedback was provided by the investigator with regard to changes in loading of the exercises.

The BP group program consisted of three 5 -week mesocycles with the first block characterized by a high training volume and low intensity, focused on muscle hypertrophy. During this training 
Table 1. Exercises for both BP (block periodization) and WUP (weekly undulating periodization) training programs

\begin{tabular}{llll}
\hline Monday & Tuesday & Thursday & Friday \\
\hline Bench press & Prone barbell row & Squat & Inclined bench press \\
Dumbell bench press & Lat machine & Leg press & Low row \\
Dips & Pull up & Leg extension / leg curl & Prone lateral lifts \\
Skull crushers & Dumbell row & Military press & Inclined dumbell curl \\
Triceps pull down & Standing barbell curl & Upright row & Preacher curl \\
& Preacher curl & Lateral lift & \\
\hline
\end{tabular}

phase participants used workloads ranging between 65 and $75 \%$ of $1 \mathrm{RM}(8-10 \mathrm{RM})$. In the subsequent mesocycles the focus transitioned to strength and power. During the strength mesocycle, subjects used $85-95 \%$ of $1 \mathrm{RM}$ loads with low repetitions (1-5 RM) and with a 3-minute recovery between sets. In the third mesocycle, athletes used workloads ranging between $50-65 \%$ of $1 \mathrm{RM}$ with maximal contraction speed; the training focus transitioned to maximal power. During the power training sessions a recovery time of three minutes between sets was used. In addition, there were 3-minute recoveries between each exercise, and a 24-hour recovery period was provided between each training session in both programs, BP and WUP.

The WUP program also consisted of three 5 -week mesocycles with decreasing training volume and increasing intensity. Each mesocycle progressed from hypertrophy to strength to a power focus with a gradual reduction in the loads used. In the first week of each mesocycle, athletes performed five sets of 8-10 reps at 65-75 $\%$ of 1 RM with a one-minute recovery between each set and exercise. In the second week participants performed five sets of 5-6 reps at $75-85 \%$ of $1 \mathrm{RM}$ with a 2-minute recovery between each set and exercise. During the third week of each mesocycle participants completed five sets of 3-4 reps at $85-95 \%$ of $1 \mathrm{RM}$ with a 3 -minute recovery between each set and exercise. In the fourth week of each mesocycle, training was focused on muscle power and the workload used was $50-60 \%$ of $1 \mathrm{RM}$ with a 3 -minute recovery between sets and exercises. An overall view shows that for both groups the training volume was gradually reduced from the beginning to the end of training program. The fifth week of each mesocycle in both training programs was used as a recovery week. Participants performed four sets of six reps with $50 \%$ of $1 \mathrm{RM}$ with a 2 -minute recovery between sets.

\section{Saliva collection and analysis}

Saliva samples were collected before and immediately after each mesocycle (which corresponded to weeks 1, 4, 6, 9, 11 and 14). Saliva sampling was conducted before and after the workout in each assessment. Each assessment corresponded to the first and last workout of each mesocycle. Two workouts were focused on muscle hypertrophy (HYP) and participants performed six sets of 10 repetitions at $70 \%$ of $1 \mathrm{RM}$ with a 1-minute recovery between sets. Two training sessions used a maximal strength (MS) scheme in which participants completed five sets of three repetitions at $90 \%$ of $1 \mathrm{RM}$ with a 3 -minute recovery between sets. Two workouts were focused on power training (PT) and five sets of six reps at $55 \%$ of $1 \mathrm{RM}$ with an explosive intent were performed with a 3-minute recovery between sets. All training protocols consisted of four exercises executed using free weights and performed in the following order: squat, bench press, inclined bench press, low barbell row. All participants performed each training session at the same time of the day, starting workouts at 4 p.m. Participants were instructed to avoid foods and liquids (except for water) for two hours before biological sampling. Saliva was collected directly into sterile tubes and no oral stimulants were used. Samples were stored at $-20^{\circ} \mathrm{C}$ until analysis. During training sessions, participants were allowed to drink water ad libitum.

Before the analysis, samples were centrifuged at $1100 \mathrm{rpm}$ for eight minutes at $6^{\circ} \mathrm{C}$. Salivary cortisol and testosterone were measured by enzyme-linked immunosorbent assay (ELISA) using commercial kits from DRG (Marburg, Germany). All samples were assayed in duplicate, using $100 \mu 1$ assays. To eliminate inter-assay variance all samples for a particular assay were analyzed in the same assay run by a single technician. Intra-assay coefficients of variations (CVs) were $2.61 \%$ and $8.16 \%$ for cortisol and testosterone, respectively. Plates were read by means of a Wallace Victor2, 1420 Multilabel Counter (Perkin-Elmer, Boston, MA).

\section{Anthropometric assessments and body composition}

Anthropometric assessments and body composition were determined prior to (PRE) and following (POST) the 15-week training program. Anthropometric measurements were assessed prior to strength assessments and included body height, body mass and body fat composition. Body mass was measured to the nearest $0.1 \mathrm{~kg}$. Body fat percentage was estimated using subcutaneous skinfold measurements at seven different sites (Jackson \& Pollock, 1978). The same qualified investigators performed all of the skinfold analysis assessments during each assessment period. The intraclass coefficient of 
variation for the investigator's skinfold measurements was 0.99 .

\section{Strength testing}

All participants were assessed for strength of the upper and lower body prior to (PRE) and following (POST) the 15-week training program. Participants completed a 5-minute submaximal warm-up on a cycle ergometer, cycling at $70 \mathrm{rpm}$ at an intensity of $50 \mathrm{~W}$. During each testing session they performed a maximal effort isometric half squat using an isometric dynamometer (Globus Iso Control, By Globus Inc., Treviso, Italy, sampling rate: $500 \mathrm{HZ}$ ). The isometric half squat test was conducted using a Smith Machine with a fixed barbell and the knee flexion angle of $90^{\circ}$ measured with a manual goniometer. Each participant performed two isometric squats with three minutes of recovery between the attempts. In addition, maximal dynamic strength of the upper body was assessed by a 1RM bench press using a Smith Machine. Bench press testing was performed in the standard supine position. The participant lowered the bar to his mid chest and then pressed the weight until his arms were fully extended. The 1RM test was conducted as previously described by Hoffman (2014). Intraclass coefficients of variation, for pre- and post-training results were 0.92 (SEM: $5.0 \mathrm{~kg}$ ) and 0.95 (SEM: 7.19 $\mathrm{kg}$ ) for the 1RM bench press and squat maximal isometric strength, respectively.

\section{Statistical analysis}

A Shapiro-Wilk test was used to assess the normality of data distribution. Data were statistically analyzed using a 2-way (group x time) analysis of variance with repeated measures for anthropometric and performance measures. A repeated measures analysis of variance (ANOVA) was used to compare mean serum salivary concentrations of testosterone and cortisol between PRE and POST training sessions for each group. If significant interactions between the different training sessions on salivary hormones were found, paired sample $t$-tests with Bonferroni adjustment were used as post-hoc. For effect size (ES), the partial eta squared statistics was reported and according to Stevens (2002), 0.01, 0.06 , and 0.14 represented small, medium, and large effect sizes, respectively. The statistical significance was accepted at $\mathrm{p} \leq .05$. Where appropriate, percent change was calculated as follows: (posttest mean pretest mean $) /($ pretest mean $) \times 100$.

\section{Results}

Anthropometric and strength performances measures of BP and WUP can be seen in Table 2. A significant main effect of time was observed for both the squat maximal isometric strength $\left(\mathrm{F}_{1,17}=13.46\right.$; $\left.\mathrm{p}=.002 ; \eta^{2}=.46\right)$ and $1 \mathrm{RM}$ bench press $\left(\mathrm{F}_{1,17}=9.40\right.$; $\left.\mathrm{p}=.007 ; \eta^{2}=.37\right)$. A significant interaction between the two groups was found for 1RM bench press $(\mathrm{p}=.040)$. A percentage increase in this parameter was higher in BP group (+7.47\%) as compared to WUP group $(+1.28 \%)$. No significant interactions between the two groups were observed for squat isometric strength $(\mathrm{p}=.168)$. Significant increments in LBM $\left(\mathrm{F}_{1,17}=25.04 ; \mathrm{p}<.001 ; \eta^{2}=0.61\right)$ and in body weight $\left(\mathrm{F}_{1,17}=7.97 ; \mathrm{p}=.025 ; \eta^{2}=.275\right)$ were observed in both groups, but no between group differences were seen ( $\mathrm{p}=.344$ and $\mathrm{p}=.288$, respectively).

Changes in salivary $\mathrm{T}$ and $\mathrm{C}$ can be observed in Tables 3 and 4, respectively. Repeated measures ANOVA revealed no significant changes in the PRE T $(\mathrm{p}=.867)$ and $\mathrm{C}(\mathrm{p}=.563)$ concentrations measured before the training sessions. When the training sessions were split by the protocol used, a significant main effect of time for salivary testosterone from the change between PRE to POST was identified $\left(\mathrm{F}_{1,101}=8.82 ; \mathrm{p}=.004 ; \eta^{2}=.080\right)$. A significant interaction between the training schemes was detected $\left(\mathrm{F}_{1,101}=3.89 ; \mathrm{p}=.024 ; \eta^{2}=.072\right)$, without significant differences between BP and WUP group $\left(\mathrm{F}_{1,101}=1.037 ; \mathrm{p}=.575 ; \eta^{2}=.026\right)$. Paired sample t-tests revealed that the increase in salivary testosterone from PRE to POST training session was significantly greater using the power training protocol (PT) compared to the maximal strength protocol (MS; $+19.0 \% ; \mathrm{p}=.007)$ and to the hypertrophy protocol (HYP; $+21.3 \%$; $=.025$ ). Changes in T concentrations in correspondence to the different training schemes can be seen in Figure 1.

Table 2. PRE to POST comparisons in strength assessments and anthropometric measures

\begin{tabular}{lcccc}
\hline & \multicolumn{2}{c}{ BP group (mean \pm SD) } & \multicolumn{2}{c}{ WUP group (mean \pm SD) } \\
Assesments & PRE & POST & PRE & POST \\
\hline Bench press 1RM (kg) & $107.0 \pm 18.8$ & $115.0 \pm 18.4^{* \wedge}$ & $97.5 \pm 17.5$ & $98.75 \pm 17.2$ \\
Maximal isom squat (kg) & $141.7 \pm 32.1$ & $146.9 \pm 36.4^{*}$ & $117.98 \pm 30.2$ & $129.95 \pm 34.5^{\star}$ \\
LBM (kg) & $71.98 \pm 7.6$ & $73.78 \pm 7.5^{*}$ & $69.40 \pm 8.8$ & $70.61 \pm 9.4^{*}$ \\
Body mass (kg) & $79.66 \pm 10.6$ & $81.02 \pm 10.2^{*}$ & $78.89 \pm 14.9$ & $79.41 \pm 14.5^{*}$ \\
FM (kg) & $7.67 \pm 3.6$ & $7.24 \pm 3.8$ & $9.48 \pm 6.8$ & $8.8 \pm 5.9$ \\
\hline
\end{tabular}

Note: * indicates a significant change from PRE to POST $(p<.05) .{ }^{\wedge}$ indicates a significant interaction between groups $(p<0.05)$. BP = block periodization; $\mathrm{WUP}=$ weekly undulating periodization; $\mathrm{LBM}=$ lean body mass; $\mathrm{FM}=$ fat mass . 
Table 3. Changes in salivary testosterone levels (mmol/L) from PRE to POST each training session

\begin{tabular}{|c|c|c|c|c|}
\hline \multirow[b]{2}{*}{ Time } & \multicolumn{2}{|c|}{ BP group (mean $\pm S D$ ) } & \multicolumn{2}{|c|}{ WUP group (mean \pm SD) } \\
\hline & $P R E$ & POST & $P R E$ & POST \\
\hline T1 & (HYP)234.22 2143.7 & $209.23 \pm 126.6$ & (HYP)233.14 \pm 112.1 & $235.90 \pm 114.0$ \\
\hline T2 & (HYP) 179.31 \pm 78.5 & $198.98 \pm 93.5$ & (MS) $214.73 \pm 72.2$ & $235.96 \pm 90.0$ \\
\hline T3 & (MS) $225.43 \pm 130.7$ & $237.75 \pm 158.7$ & (HYP) $179.63 \pm 59.6$ & $208.78 \pm 91.9$ \\
\hline T4 & (MS) $214.29 \pm 94.8$ & $207.02 \pm 132.7$ & (PT) $198.64 \pm 46.3$ & $242.66 \pm 103.3$ \\
\hline T5 & (PT) $221.56 \pm 108.8$ & $269.62 \pm 136.8$ & (MS) $205.17 \pm 67.2$ & $224.63 \pm 87.6$ \\
\hline T6 & (PT) $210.14 \pm 130.5$ & $276.80 \pm 162.2$ & (PT) $255.99 \pm 91.8$ & $302.39 \pm 83.3$ \\
\hline
\end{tabular}

Note: BP: block periodization; WUP: weekly undulating periodization. HYP: hypertrophy scheme; MS: maximal strength scheme; PT: power training scheme.

No main effects for time $\left(\mathrm{F}_{1,86}=.721\right.$; $\left.\mathrm{p}=.334 ; \eta^{2}=.008\right)$ and no interaction were seen between the groups $\left(\mathrm{F}_{1,86}=.102 ; \mathrm{p}=.633\right.$; $\left.\eta^{2}=.001\right)$ for salivary $\mathrm{C}$ concentrations. In addition, there were no PRE to POST differences between the HYP, MS and PT protocols $\left(\mathrm{F}_{1,86}=7.929 ; \mathrm{p}=.093 ; \eta^{2}=.054\right)$.

\section{Discussion and conclusions}

The primary purpose of this study was to compare endocrine and strength adaptations during 15-week weekly undulating or block periodized resistance training programs in experienced resistance trained men. Results of the present study showed that the BP model led to greater improvements in $1 \mathrm{RM}$ bench press strength than the WUP program. However, no significant differences were observed between the training models in lower body maximal isometric strength and lean body mass gains. Despite significant increases were noted in lower body maximal isometric strength in both groups, performing leg training once a week may not be optimal to stimulate further adaptations in trained men. Specifically, the BP group increased $1 \mathrm{RM}$ bench press by an average of $7.47 \%$, whereas the WUP group increased bench press maximal strength by an average of $1.28 \%$. These increments, detected in upper body strength, are consistent with the findings of others (Apel, Lacey, \& Kell, 2011; Bartolomei, et al., 2014; Hoffman, et al., 2003) who suggested that a training program composed of mesocycles and characterized by a relatively constant volume and intensity was more effective in eliciting strength gains than undulating type periodized programs. The benefits observed in the block periodization model may be related to the timing of the workload and its distribution. According to Issurin (2010), concentrated training stimuli may have optimized the supercompensation process and produced a cumulative effect of different training loads.

Neither training program appeared to significantly affect basal testosterone levels across the

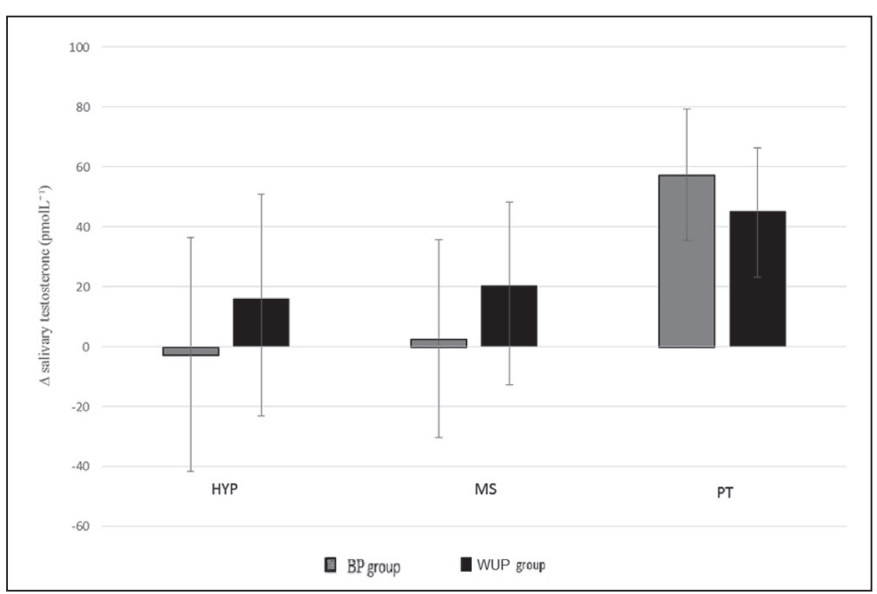

Figure 1. Differences in testosterone concentrations between PRE and POST each training schemes. HYP: hypertrophy protocol; MS: maximal strength protocol; PT: power training protocol.

training cycles. This is consistent with previous research that reported prolonged resistance training had minimal effects on the circadian pattern of salivary testosterone (Kraemer, et al., 2001). This is also consistent with other studies that have reported no changes in resting concentrations of testosterone during training programs of a similar durations (Hoffman, Ratamess, Kang, Falvo, \& Faingenbaum, 2006, 2007) or lasting longer (Häkkinen, Pakarinen, Alén, Kauhanen, \& Komi, 1987).

No changes were noted in resting cortisol concentrations in either group during this 15 -week training study. This is consistent with some studies (Hoffman, et al., 2006, Potteiger, Judge, Cerny, \& Potteiger, 1995), but in contrast with others (Fry, et al. 1992; Häkkinen, \& Pakarinen, 1991; Hoffman, et al., 2007). The variability in the cortisol response to training may reflect various psychological and physiological stresses that can impact cortisol secretion patterns. It is often used to denote training stress (Fry \& Kraemer, 1997; Fry, Kraemer, \& Ramsey, $1998)$ and lack of any change may reflect a positive training adaptation. The persistence of cortisol concentrations in both training protocols suggest that both protocols were performed in a manner that minimized physiological stress. The absence of 
chronic hormonal variations in both BP and WUP group may be related to the inclusion of a limited number of lower body strength exercises and to the lack of Olympic lifts and their variations in the training program.

The results of this study also indicated that the acute testosterone response to exercise was dependent upon the training phase. Regardless of the periodization model used, participants exhibited significantly elevated testosterone responses to the exercise routine during the power phase. No changes were noted in the salivary response of testosterone and cortisol to either the strength or hypertrophy phases. This appears to be in contrast to several studies that reported significant elevations in both salivary and serum testosterone concentrations following high-volume hypertrophy protocols with no significant changes during power and strength workouts (Crewther, et al., 2008; Kraemer, et al., 1991; Linnamo, Pakarinen, Komi, Kraemer, \& Häkkinen, 2005; Smilios, Tsoukos, Zafeiridis, Spassis, \& Tokmakidis, 2014). These studies suggest that a relative intensity and volume threshold should be reached in order to stimulate a testosterone response. Studies that examined the acute hormonal response to resistance exercise have generally focused on traditional movements using heavy loads at a relatively slow velocity (Fry \& Lohnes, 2010). A few investigations have examined the effects of power training sessions incorporating lighter loads and higher velocities, and some of them found significant effects of explosive power training on circulating testosterone (Fry \& Lohnes,
2010; Volek, Kraemer, Bush, Incledon, \& Boetos, 1997). Findings from the present study support the notion that speed of contraction may be an important stimulus for testosterone secretion. The execution of movements at maximum velocity requires the activation of a large muscle mass during the early stages of exercise. An increased rate of power development may induce hormonal responses as a result of neural and muscular mechanisms (Fry, et al., 1998). Increases in salivary testosterone may be related to a high psychophysical activation essential to activate a high number of motor units during high power muscle contractions (Salvador, Suay, González-Bono, \& Serrano, 2003) and, consistent with Issurin (2010), may be also influenced by the cumulative effects of the hypertrophy and maximal strength phases.

This investigation demonstrated that block periodization may be more effective than the weekly undulating periodization model in increasing upper body strength in experienced, resistance trained males. No differences between the two training programs have been observed for lower body strength gains. In addition, changes in the testosterone response suggest the potential anabolic response associated with the power mesocycle during a periodized resistance training program in trained athletes. Professionals concerned with designing optimal training programs for strength and power athletes should be aware that the introduction of sessions focused on muscle power may optimize the body adaptation as a result of testosterone elevation.

\section{References}

Apel, J.M., Lacey, R.M., \& Kell, R.T. (2011). A comparison of traditional and weekly undulating periodized strength training programs with total volume and intensity equated. Journal of Strength and Conditioning Research, 25(3), 694-703.

Bartolomei, S., Hoffman, J.R., Merni F., \& Stout J.R. (2014). A comparison of traditional and block periodized strength training programs in trained athletes. Journal of Strength and Conditioning Research, 25(8), 990-997.

Bartolomei, S, Stout, J.R., Fukuda, H.D., Hoffman, J.R., \& Merni, F. (2015). Block versus weekly undulating periodized resistance training programs in women. Journal of Strength and Conditioning Research, 29(10), 2679-2687.

Beaven, C.M., Gill, N.D., \& Cook, C.J. (2008). Salivary testosterone and cortisol responses in professional rugby players after four resistance exercise protocols. Journal of Strength and Conditioning Research, 22(2), 426-432.

Buford, T.W., Rossi S.J., Smith, D.B., \& Warren, A.J. (2007). A comparison of periodization models during nine weeks with equated volume and intensity for strength. Journal of Strength and Conditioning Research, 21(4), 1245-1250.

Cadore, L.E., Lhullier, F.L., Arias Brentano, M., Marczwski Da Silva, E., Bueno Ambrosini, M., Spinelli, R., et al. (2009). Salivary hormonal responses to resistance exercise in trained and untrained middle-aged men. Journal of Sports Medicine and Physical Fitness, 49(3), 301-307.

Crewther, B.T., Cronin, J., Keogh, J., \& Cook, C. (2008). The salivary testosterone and cortisol response to three loading schemes. Journal of Strength and Conditioning Research, 22(1), 250-255.

Crewther, B.T., Lowe, T.E., Ingram, J., \& Weatherby, R.P. (2010). Validating the salivary testosterone and cortisol concentration measures in response to short high-intensity exercise. Journal of Sports Medicine and Physical Fitness, 50(1), 85-92.

Fry, A.C., \& Kraemer, W.J. (1997). Resistance exercise overtraining and overreaching: Neuroendocrine response. Sports Medicine, 23(2), 106-129. 
Fry, A.C., Kraemer, W.J., \& Ramsey, L.T. (1998). Pituitary-adrenal-gonadal responses to high-intensity resistance exercise overtraining. Journal of Applied Physiology, 85(6), 2352-2359.

Fry, A.C., Kraemer, W.J., Stone, M.H., Warren, B., Kearney, J.T., Fleck, S.J., et al. (1992). The effect of amino acid supplementation on testosterone, cortisol, and growth hormone responses to one week of intensive weightlifting. International Journal of Sport Nutrition and Exercise Metabolism, 3, 306-322.

Fry, A.C., \& Lohnes, C.A. (2010). Acute testosterone and cortisol responses to high power resistance training. Human Physiology, 36(4), 457-461.

Häkkinen, K., \& Pakarinen, A. (1991). Serum hormones in male strength athletes during intensive short term strength training. European Journal of Applied Physiology, 63(3-4), 194-199.

Häkkinen, K., Pakarinen, A., Alén, M., Kauhanen, H., \& Komi, P.V. (1987). Relationship between training volume, physical performance capacity, and serum hormone concentrations during prolonged training in elite weight lifters. International Journal of Sports Medicine, 8, 61-65.

Hoffman, J.R. (2014). Physiological aspects of sport training and performance (2 ${ }^{\text {nd }}$ ed.). Champaign, IL: Human Kinetics.

Hoffman, J.R., Ratamess, N.A., Kang, J., Falvo, M.J., \& Faingenbaum, A.D. (2006). Effects of protein intake on strength, body composition and endocrine changes in strength/power athletes. Journal of the International Society of Sport Nutrition, 3(2), 12-18.

Hoffman, J.R., Ratamess, N.A., Kang, J., Falvo, M.J., \& Faingenbaum, A.D. (2007). Effects of protein supplementation on muscular performance and resting hormonal changes in college football players. Journal of Sports Science and Medicine, 6(1), 85-92.

Hoffman, J.R., Wendell, M., Cooper, J., \& Kang, J. (2003). Comparison between linear and nonlinear in-season training programs in freshman football players. Journal of Strength and Conditioning Research, 17(3), 561-565.

Issurin, V.B. (2010). New horizons for the methodology and physiology of training periodization. Sports Medicine, 40(3), 189-206.

Jackson, A.S., \& Pollock, M.L. (1978). Generalized equations for predicting body density of men. British Journal of Nutrition, 40(3), 497-504.

Kraemer, W.J., Gordon, S.E., Fleck, S.J., Marchitelli, L.J., Mello, R., Dziados, J.E., et al. (1991). Endogenous anabolic hormonal and growth factor responses to heavy resistance exercise in males and females. International Journal of Sports Medicine, 12(2), 228-235.

Kraemer, W.J., Loebel, C.C., Volek, J.S., Ratamess, N.A., Newton, R.U., Wickam, R.B, et al. (2001). The effect of heavy resistance exercise on the circadian rhythm of salivary testosterone in men. European Journal of Applied Physiology, 84(1-2),13-18.

Linnamo, V., Pakarinen, A., Komi, P.V., Kraemer, W.J., \& Häkkinen, K. (2005). Hormonal responses to submaximal and maximal heavy resistance and explosive exercises in men and women. Journal of Strength and Conditioning Research, 19(3), 566-571.

Matveev, L.P. (1965). The problem of training periodization in sports. [In Russian.] Moscow: Progress Publishers.

Matveev, L. (1977). Fundamentals of sport training. [A.P. Zdornykh, Trans.] Moscow: Fizkultura i Sport.

McGuigan, M.R., Egan, A.D., \& Foster, C. (2004). Salivary cortisol responses and perceived exertion during high intensity and low intensity bouts of resistance exercise. Journal of Sports Science and Medicine, 3(1), 8-15.

Nádori, L., \& Granek, I. (1989). Theoretical and methodological basis of training planning with special considerations within a microcycle. Lincoln, NE: National Strength and Conditioning Association.

Poliquin, C. (1988). Five steps to increasing the effectiveness of your strength training program. National Strength and Conditioning Association Journal, 10(3), 34-37.

Potteiger, J., Judge, L., Cerny, J., \& Potteiger, V. (1995). Effects of altering training volume and intensity on body mass, performance and hormonal concentrations in weight-events athletes. Journal of Strength and Conditioning Research, 9(1), 55-58.

Salvador, A., Suay, F., González-Bono, E., \& Serrano, M.A. (2003). Anticipatory cortisol, testosterone and psychological responses to judo competition in young men. Psychoneuroendocrinology, 28(3), 364-375.

Smilios, I., Tsoukos, P., Zafeiridis, A., Spassis, A., \& Tokmakidis, S.P. (2014). Hormonal responses after resistance exercise performed with maximal and submaximal movement velocities. Applied Physiology, Nutrition and Metabolism, 39(3), 351-357.

Stevens, J.P. (2002). Applied multivariate statistics for the social science. Mahaw, NJ: Lawrence Erlbaum Associates.

Stone, M.H., Potteiger, J.A., Pierce, K.C., Proulx, C.M., O’Bryant, H.S., Johson, R.L., et al. (2000). A comparison of the effect of three different weight-training programs on the one repetition maximum squat. Journal of Strength and Conditioning Research, 14(3), 332-337.

Umeda, T., Hiramatsu, R., Iwaoka, T., Shimada, T., Miura, F., \& Sato, T. (1981). Use of saliva for monitoring unbound free cortisol levels in serum. Clinica Chimica Acta, 110, 245-253.

Verkhoshansky, J.V. (1977). Fundamentals of special strength training in sport. [A.C. Charniga, Trans.] Moscow: Fizkultura i Sport.

Verkhoshansky, J.V. (2001). La moderna programmazione dell'allenamento sportivo. [O. Jourtchennko, Trans.] Roma: Scuola dello Sport. 
Vittek, J., L'Hommedieu, D.G., Gordon, G.G., Rappaport, S.C., \& Southren, A. (1985). Direct radioimmunoassay (RIA) of salivary testosterone: Correlation with free and total serum testosterone. Life Sciences, 37(8), 711-716.

Volek, J.S., Kraemer, W.J., Bush, J.A., Incledon, T., \& Boetes, M. (1997). Testosterone and cortisol in relationship to dietary nutrients and resistance exercise. Journal of Applied Physiology, 82(1), $49-54$.

Zatsiorsky, V.M., \& Kraemer, W.J. (2006). Science and practice of strength training ( $2^{\text {nd }}$ ed.). Champaign, IL: Human Kinetics.

Submitted: February 17, 2016

Accepted: April 21, 2016

Correspondence to:

Sandro Bartolomei, Ph. D.

University of Central Florida

4000 Central Florida Boulevard

Orlando 32816, USA

Phone: 1-407-8232367

E-mail: sandro.bartolomei@ucf.edu

\section{Aknowledgements}

C. Stefanelli thanks the "Fondazione del Monte di Bologna e Ravenna" for financial support. 